\title{
Some Combinatorial Characterizations of Gorenstein Graphs with Independence Number Less than Four
}

\author{
Mohammad Reza Oboudi, Ashkan Nikseresht* \\ Department of Mathematics, College of Sciences, Shiraz University \\ 71457-44776, Shiraz, Iran \\ E-mail:mr_oboudi@yahoo.com andmr_oboudi@shirazu.ac.ir \\ E-mail: a_nikseresht@shirazu.ac.ir
}

\section{Abstract}

Let $\alpha=\alpha(G)$ be the independence number of a simple graph $G$ with $n$ vertices and $I(G)$ be its edge ideal in $S=K\left[x_{1}, \ldots, x_{n}\right]$. If $S / I(G)$ is Gorenstein, the graph $G$ is called Gorenstein over $K$ and if $G$ is Gorenstein over every field, then we simply say that $G$ is Gorenstein. In this article, first we state a condition equivalent to $G$ being Gorenstein and using this we give a characterization of Gorenstein graphs with $\alpha=2$. Then we present some properties of Gorenstein graphs with $\alpha=3$ and as an application of these results we characterize triangle-free Gorenstein graphs with $\alpha=3$.

Keywords: Gorenstein ring; Edge ideal; Triangle-free graph; Independence polynomial.

2010 Mathematical Subject Classification: 13F55, 05E40, $13 \mathrm{H} 10$.

\footnotetext{
${ }^{*}$ Corresponding author
}

\section{Introduction}

Throughout this paper, $K$ is a field, $S=$ $K\left[x_{1}, \ldots, x_{n}\right]$ and $G$ denotes a simple undirected graph with vertex set $\mathrm{V}(G)=$ $\left\{v_{1}, \ldots, v_{n}\right\}$ and edge set $\mathrm{E}(G)$. Recall that the edge ideal $I(G)$ of $G$ is the ideal of $S$ generated by $\left\{x_{i} x_{j} \mid v_{i} v_{j} \in \mathrm{E}(G)\right\}$. Many researchers have studied how algebraic properties of $S / I(G)$ relates to the combinatorial properties of $G$ (see [2-4, 13] and references therein). An important algebraic property that recently has gained attention is being Gorenstein. Recall that Gorenstein rings and Cohen-Macaulay rings (CM rings for short) are central concepts in commutative algebra. We refer the reader to [1] for their definitions and basic properties. We say that $G$ is a Gorenstein (resp. Cohen-Macaulay) graph over $K$, if $S / I(G)$ is a Gorenstein (resp. CM) ring. When $G$ is Gorenstein (resp. CM) over every field, we say that $G$ is Gorenstein (resp. $\mathrm{CM})$. In [3] and [4] characterizations of pla- 
nar Gorenstein graphs of girth at least four and triangle-free Gorenstein graphs are presented, respectively. (Recall that $G$ is said to be triangle-free when no subgraph of $G$ is a triangle). Also in [13] a condition on a planar graph equivalent to being Gorenstein is stated and proved. An importance of characterizing Gorenstein graphs comes from the Charney-Davis conjecture which states that "flag simplicial complexes" which are Gorenstein over $\mathbb{Q}$ satisfy a certain condition (see [10, Problem 4]). In [10], Richard P. Stanley mentioned this conjecture as one of the "outstanding open problems in algebraic combinatorics" at the start of the $21^{\text {st }}$ century. An approach to solve this conjecture is trying to give a characterization of Gorenstein "flag simplicial complexes" which is equivalent to a characterization of Gorenstein graphs by [2, Lemma 9.1.3].

Denote by $\alpha(G)$ the independence number of $G$, that is, the maximum size of an independent set of $G$. It is well-known that if $\alpha(G)=1$ (that is, $G$ is complete) then $G$ is Gorenstein if and only if $G=K_{1}$ or $G=K_{2}$ (where $K_{n}$ denotes the complete graph on $n$ vertices). Moreover, it is easy to see that if $\alpha(G)=2$, then $G$ is Gorenstein if and only if $G$ is the complement of a cycle of length at least four (see Corollary 2.5 below). Therefore, it is natural to ask "if $\alpha(G)=3$, then when is $G$ Gorenstein?" In this paper, we present some properties of Gorenstein graphs with $\alpha=3$ and find all Gorenstein graphs with $\alpha=3$ which are also triangle-free. Before stating our main results and since we need some results on Gorenstein simplicial complexes, first we briefly review simplicial complexes and their Stanley-Reisner ideal.

Recall that a simplicial complex $\Delta$ on the vertex set $V=\mathrm{V}(\Delta)=\left\{v_{1}, \ldots, v_{n}\right\}$ is a family of subsets of $V$ (called faces) with the property that $\left\{v_{i}\right\} \in \Delta$ for each $i \in[n]=$ $\{1, \ldots, n\}$ and if $A \subseteq B \in \Delta$, then $A \in \Delta$. In the sequel, $\Delta$ always denotes a simplicial complex. Thus the family $\Delta(G)$ of all cliques of a graph $G$ is a simplicial complex called the clique complex of $G$. Also $\Delta(\bar{G})$ is called the independence complex of $G$, where $\bar{G}$ denotes the complement of $G$. Note that the elements of $\Delta(\bar{G})$ are independent sets of $G$. The ideal of $S$ generated by $\left\{\prod_{v_{i} \in F} x_{i} \mid F \subseteq V\right.$ is a non-face of $\Delta\}$ is called the StanleyReisner ideal of $\Delta$ and is denoted by $I_{\Delta}$ and $S / I_{\Delta}$ is called the Stanley-Reisner algebra of $\Delta$ over $K$. Therefore we have $I_{\Delta(\bar{G})}=I(G)$. If the Stanley-Reisner algebra of $\Delta$ over $K$ is Gorenstein (resp. CM), then $\Delta$ is called Gorenstein (resp. CM) over $K$. The relation between combinatorial properties of $\Delta$ and algebraic properties of $S / I_{\Delta}$ is well-studied, see for example $[2,6,8,9,12]$ and the references therein.

The dimension of a face $F$ of $\Delta$ and the simplicial complex $\Delta$ are defined to be $|F|-$ 1 and $\max \{\operatorname{dim}(F) \mid F \in \Delta\}$, respectively. Recall that $\alpha(G)$ denotes the independence number of $G$, that is, $\alpha(G)=\operatorname{dim} \Delta(\bar{G})+1$. A graph $G$ is called well-covered, if all maximal independent sets of $G$ have size $\alpha(G)$ and we say that $G$ is a $W_{2}$ graph, if $|\mathrm{V}(G)| \geq 2$ and every pair of disjoint independent sets of $G$ are contained in two disjoint maximum independent sets. In some texts, $\mathrm{W}_{2}$ graphs are called 1-well-covered graphs. The reader can see [11] for more on $W_{2}$ graphs. In the fol- 
lowing lemma, we have collected two known results on $W_{2}$ graphs and their relation to Gorenstein graphs.

Lemma 1.1. (i) ([3, Lemma 3.1] or [4, Lemma 3.5]) If $G$ is a graph without isolated vertices and $G$ is Gorenstein over some field $K$, then $G$ is a $W_{2}$ graph.

(ii) ([4, Proposition 3.7]) If $G$ is triangle-free and without isolated vertices, then $G$ is Gorenstein if and only if $G$ is $W_{2}$.

Let $f_{i}$ be the number of $i$-dimensional faces of $\Delta$ (if $\Delta \neq \emptyset$, we set $f_{-1}=1$ ), then $\left(f_{-1}, \ldots, f_{d-1}\right)$ is called the $f$-vector of $\Delta$, where $d-1=\operatorname{dim}(\Delta)$. Now define $h_{i}$ 's such that $h(t)=\sum_{i=0}^{d} h_{i} t^{i}=\sum_{i=0}^{d} f_{i-1} t^{i}(1-$ $t)^{d-i}$. Then $h(t)$ is called the $h$-polynomial of $\Delta$. Recall that the polynomial $I(G, x)=$ $\sum_{i=0}^{\alpha(G)} a_{i} x^{i}$, where $a_{i}$ is the number of independent sets of size $i$ in $G$ and $a_{0}=1$, is called the independence polynomial of $G$. Note that $\left(a_{0}, a_{1}, \ldots, a_{\alpha(G)}\right)$ is the $f$-vector of $\Delta(\bar{G})$. There are many papers related to this polynomial in the literature, see for example [5].

For $F \in \Delta$, let $\operatorname{link}_{\Delta}(F)=\{A \backslash F \mid F \subseteq$ $A \in \Delta\}$. If all maximal faces of $\Delta$ have the same dimension and for each $F \in \Delta$, we have $\sum_{i=-1}^{d_{F}}(-1)^{i} f_{i}(F)=(-1)^{d_{F}}(*)$, where $d_{F}=\operatorname{dim}_{\operatorname{link}_{\Delta}}(F)$ and $\left(f_{i}(F)\right)_{i=-1}^{d_{F}}$ is the $f$-vector of $\operatorname{link}_{\Delta}(F)$, then $\Delta$ is said to be an Euler complex. Another complex constructed from $\Delta$ is core $(\Delta)$. The set of vertices of $\operatorname{core}(\Delta)$ is $V_{C}=\{v \in \mathrm{V}(\Delta) \mid \exists G \in$ $\Delta$ such that $G \cup\{v\} \notin \Delta\}$ and $\operatorname{core}(\Delta)=$ $\left\{F \in \Delta \mid F \subseteq V_{C}\right\}$.

\section{Main results}

First we establish a theorem which presents a condition on graphs equivalent to being Gorenstein. For this, we need the following lemma.

Lemma 2.1. If $G$ is a graph without any isolated vertex and Gorenstein over some field $K$, then $I(G,-1)=(-1)^{\alpha(G)}$.

Proof. Let $\Delta=\Delta(\bar{G})$ and $d=\operatorname{dim} \Delta=$ $\alpha(G)-1$. If $v \in \mathrm{V}(G)$, then as $v$ is not isolated, there is a $u \in \mathrm{V}(G)$ such that $u v \in$ $\mathrm{E}(G)$. Hence $G=\{u\} \in \Delta$ and $G \cup\{v\} \notin \Delta$. This means that $v \in \operatorname{core}(\Delta)$ and hence $\operatorname{core}(\Delta)=\Delta$. So according to $[1$, Theorem 5.5.2], $\Delta$ is an Euler complex. In particular, noting that $\operatorname{link}_{\Delta}(\emptyset)=\Delta$, if we write $(*)$ with $F=\emptyset$, we get $\sum_{i=-1}^{d}(-1)^{i} f_{i}=(-1)^{d}$ where $\left(f_{i}\right)_{i=-1}^{d}$ is the $f$-vector of $\Delta$. Thus

$$
\begin{aligned}
I(G,-1) & =\sum_{i=0}^{\alpha(G)} a_{i}(-1)^{i}=\sum_{i=0}^{\alpha(G)}(-1)^{i} f_{i-1} \\
& =-\sum_{j=-1}^{d}(-1)^{j} f_{j} \\
& =(-1)^{d+1}=(-1)^{\alpha(G)} .
\end{aligned}
$$

Remark 2.2. The above argument shows that if $G$ has no isolated vertex, then $\Delta(\bar{G})=$ $\operatorname{core}(\Delta(\bar{G}))$ and if moreover $G$ is Gorenstein over some field, then $\Delta(\bar{G})$ is an Euler complex.

Suppose that $F \subseteq \mathrm{V}(G)$. Here by $\mathrm{N}[F]$ we mean $F \cup\{v \in \mathrm{V}(G) \mid u v \in \mathrm{E}(G)$ for some 
$u \in F\}$ and we set $G_{F}=G \backslash \mathrm{N}[F]$. In particular, for $F=\emptyset$ we have $\mathrm{N}[F]=\emptyset$ and $G_{F}=G$. Our next theorem gives an equivalent condition on graphs with $\alpha \geq 2$ for being Gorenstein. Recall that if $\alpha(G)=1$, then $G$ is Gorenstein if and only if $G$ is isomorphic to $K_{1}$ or $K_{2}$. Note that if $\Delta$ is a simplicial complex with dimension at most one, then we can view $\Delta$ as a graph on vertex set $\mathrm{V}(\Delta)$ by considering 1-dimensional faces of $\Delta$ as edges.

Theorem 2.3. Suppose that $G$ is a graph without any isolated vertex and $\alpha(G) \geq 2$. Then $G$ is Gorenstein over $K$ if and only if all of the following conditions hold:

(i) $G$ is $C M$ over $K$;

(ii) $I(G,-1)=(-1)^{\alpha(G)}$;

(iii) For each independent set $F$ of $G$ with size $\alpha(G)-2$, the graph $\overline{G_{F}}$ is a cycle of length at least 4.

Proof. $(\Rightarrow)$ : By Lemma 2.1, $I(G,-1)=$ $(-1)^{\alpha(G)}$ and $G$ is CM over $K$ by definition. If $G=K_{2}$, then it has no independent set of size 2 and hence (iii) holds trivially. So we assume that $G \neq K_{2}$.

Suppose that $F$ is an independent set of $G$ with size $\alpha(G)-2$. First we claim that $\overline{G_{F}}$ is not a triangle or a path of length 1 or 2. Suppose that the claim is not true. First, assume that $\overline{G_{F}}$ consists of just an edge $a b$. Let $A=F \cup\{a\}$ and $B=\{b\}$ which are two disjoint independent sets of $G$. By Lemma 1.1(i), $G$ is $\mathrm{W}_{2}$ and there exist independent sets $A_{0}$ and $B_{0}$ of size $\alpha(G)$ such that $A \subseteq A_{0}$, $B \subseteq B_{0}$ and $A_{0} \cap B_{0}=\emptyset$. But the only vertex of $G$ which is not adjacent to any vertex of $A$ in $G$ is $b$, so $A_{0}=A \cup\{b\}$. But $b \in B \subseteq B_{0}$ which contradicts $A_{0} \cap B_{0}=\emptyset$. From this contradiction it follows that $\overline{G_{F}}$ is not a path of length 1.

Now assume that $\overline{G_{F}}$ is a path of length 2 with edges $a b$ and $b c$. Let $A=\{a, b\}$ and $B=F \cup\{c\}$. Again $A$ and $B$ are disjoint independent sets of $G$ and since $G$ is $\mathrm{W}_{2}$, there exist independent sets $A_{0}$ and $B_{0}$ of size $\alpha(G)$ such that $A \subseteq A_{0}, B \subseteq B_{0}$ and $A_{0} \cap B_{0}=\emptyset$. But $b$ is the only vertex of $G$ not adjacent to any vertex of $B$. Therefore $b \in A_{0} \cap B_{0}$, which is a contradiction, thus $\overline{G_{F}}$ is not a path of length 2. Also we have $\alpha\left(G_{F}\right)=2$ and hence $\overline{G_{F}}$ can not be a triangle. This concludes the proof of the claim.

Note that because $\Delta=\Delta(\bar{G})$ contains all vertices of $G$, it is not empty and since $G \neq K_{1}, K_{2}$, its independence complex $\Delta$ does not consist of only one or two isolated vertices. Also by Remark 2.2, $\operatorname{core}(\Delta)=\Delta$. Hence by equivalence of parts (a) and (e) of [9, Chapter II, Theorem 5.1], $\operatorname{link}_{\Delta}(F)$ is either a cycle or a path of length 1 or 2 (when viewed as a graph, as mentioned in the notes before this theorem). Let $A \in \operatorname{link}_{\Delta}(F)$, then there is a $B \in \Delta$ containing $F$, such that $A=B \backslash F$. So $B=A \cup F$ is an independent set of $G$. Hence $A$ is an independent set of $G_{F}$, that is, $A \in \Delta\left(\overline{G_{F}}\right)$. Conversely, if $A \in \Delta\left(\overline{G_{F}}\right)$, then $A \cup F$ is an independent set of $G$ and hence $A \in \operatorname{link}_{\Delta}(F)$. Therefore, $\operatorname{link}_{\Delta}(F)=\Delta\left(\overline{G_{F}}\right)$. If we view this 1dimensional simplicial complex as a graph, then $\Delta\left(\overline{G_{F}}\right)=\overline{G_{F}}$. Hence $\overline{G_{F}}$ is either a cycle or a path of length 1 or 2. But as we showed above, $\overline{G_{F}}$ can not be a triangle or a path of length 1 or 2 . Consequently, $\overline{G_{F}}$ is a 
cycle of length at least 4, as required.

$(\Leftarrow)$ : Let $F$ be an independent set of size $\alpha(G)-2$ and $\Delta=\Delta(\bar{G})$. According to the argument in the above paragraph, $\operatorname{link}_{\Delta}(F)=$ $\Delta\left(\overline{G_{F}}\right)=\overline{G_{F}}$. Therefore, $\operatorname{link}_{\Delta}(F)$ is a cycle for each independent set of size $\alpha(G)-2$ of $G$. Also $\operatorname{core}(\Delta)=\Delta$ by Remark 2.2. Hence the result follows from the equivalence of (a) and (e) of [9, Chapter II, Theorem 5.1].

Remark 2.4. Note that Theorem 2.3 does not classify all Gorenstein graphs with $\alpha \geq 2$. In fact, for this we first need to classify CM graphs, which is a hard task.

As an immediate corollary of the previous theorem, we get the following characterization of Gorenstein graphs with $\alpha(G)=2$.

Corollary 2.5. Suppose that $G$ is a graph without any isolated vertex and $\alpha(G)=2$. Then the following are equivalent.

(i) G is Gorenstein over some field.

(ii) $G$ is Gorenstein over every field.

(iii) $G$ is the complement of a cycle of length at least four.

Proof. (ii) $\Rightarrow$ (i) is trivial and (i) $\Rightarrow$ (iii) follows from Theorem 2.3(iii) with $F=\emptyset$.

(iii) $\Rightarrow$ (ii): Suppose that $G$ is the complement of an $n$-cycle with $n \geq 4$. If we view $\Delta(\bar{G})$ as a graph, then $\Delta(\bar{G})$ is a cycle and hence is connected. Therefore, by $[1$, Exercise 5.1.26(c)], $\Delta(\bar{G})$ or equivalently $G$ is CM over every field. Also $I(G, x)=1+n x+n x^{2}$ and $I(G,-1)=1=(-1)^{\alpha(G)}$. Thus the result follows from Theorem 2.3.
Remark 2.6. Note that item (iii) of the above corollary determines a large class of Gorenstein graphs combinatorially.

Next we find some properties of Gorenstein graphs with $\alpha(G)=3$. For this, we need a lemma.

Lemma 2.7. Assume that $G$ is a graph without any isolated vertex and Gorenstein over a field $K$ and suppose that $\alpha(G)$ is odd, then $I\left(G,-\frac{1}{2}\right)=0$.

Proof. Let $\Delta=\Delta(\bar{G})$ and $\alpha=\alpha(G)$. By Remark 2.2, $\Delta$ is an Euler complex of dimension $\alpha-1$. Thus by [1, Theorem 5.4.2], we have $h_{i}=h_{\alpha-i}$, where $h(t)=\sum_{i=0}^{\alpha} h_{i} t^{i}=$ $\sum_{i=0}^{\alpha} f_{i-1} t^{i}(1-t)^{\alpha-i}$ is the $h$-polynomial of $\Delta$. Since $\alpha$ is odd, this means that $h(-1)=0$.

Now

$$
\begin{aligned}
I\left(G,-\frac{1}{2}\right) & =\sum_{i=0}^{\alpha} a_{i}\left(-\frac{1}{2}\right)^{i} \\
& =\sum_{i=0}^{\alpha} f_{i-1}\left(-\frac{1}{2}\right)^{i} \\
& =\frac{1}{2^{\alpha}} \sum_{i=0}^{\alpha} f_{i-1}(-1)^{i}(1-(-1))^{\alpha-i} \\
& =\frac{1}{2^{\alpha}} h(-1)=0 .
\end{aligned}
$$

Now we can prove a theorem which determines the number of edges and the independence polynomial of Gorenstein graphs with independence number three.

Theorem 2.8. Suppose that $G$ is a graph with $n$ vertices and $m$ edges and without any 
isolated vertex. Also assume that $G$ is Gorenstein over some field and $\alpha(G)=3$. Then the following hold.

(i) $m=\frac{n^{2}-7 n+12}{2}$.

(ii) $G$ has exactly $2 n-4$ independent sets of size three and exactly $3 n-6$ independent sets of size two.

(iii) $I(G, x)=1+n x+(3 n-6) x^{2}+(2 n-4) x^{3}$.

Proof. Suppose that $I(G, x)=\sum_{i=0}^{\alpha(G)} a_{i} x^{i}$ is the independence polynomial of $G$. Then $a_{0}=1, a_{1}=n$ and $a_{2}$ and $a_{3}$ are the number of independent sets of size two and three of $G$, respectively. According to Lemmas 2.1 and 2.7, $I(G,-1)=(-1)^{3}=-1$ and $I\left(G,-\frac{1}{2}\right)=0$. It follows that $a_{2}-a_{3}=n-2$ and $2 a_{2}-a_{3}=4 n-8$. Therefore $a_{2}=3 n-6$ and $a_{3}=2 n-4$, which proves (ii) and (iii). On the other hand, independent sets of size two are exactly those 2-subsets $\{u, v\}$ of $\mathrm{V}(G)$ such that $u v \notin \mathrm{E}(G)$. Thus $3 n-6=a_{2}=$ $\left(\begin{array}{l}n \\ 2\end{array}\right)-m$ and $m=\frac{n^{2}-7 n+12}{2}$.

In what follows, we apply the previous results to find all triangle-free Gorenstein graphs with $\alpha=3$. It should be mentioned that this result also follows by using [4, Theorem 4.4] and [12, Theorem 3.8], but the proof of these theorems use much algebraic background, against the following graph theoretic proof. In what follows, by $G_{v}$ we mean $G_{\{v\}}=G \backslash \mathrm{N}[v]$, where $v \in \mathrm{V}(G)$. Also $C_{n}$ denotes the $n$-vertex cycle.

Theorem 2.9. Suppose that $G$ is trianglefree, $\alpha(G)=3$ and $G$ has no isolated vertices. Then the following are equivalent: (i) $G$ is Gorenstein over every field;

(ii) G is Gorenstein over some field;

(iii) $G$ is $W_{2}$.

(iv) $G$ is isomorphic to one of the graphs depicted in Figure 1.

Proof. (i) $\Longleftrightarrow$ (iii) is an especial case of [4, Proposition 3.7] and (i) $\Rightarrow$ (ii) is trivial.

(iv) $\Rightarrow$ (iii): Let $G$ be one of the graphs in Figure 1. For each $v \in \mathrm{V}(G)$, one can check that $G \backslash v$ is well-covered and $\alpha(G \backslash$ $v)=\alpha(G)$. According to [11, Theorem 1], this means that $G$ is $\mathrm{W}_{2}$.

(ii) $\Rightarrow$ (iv): Suppose that $n=|\mathrm{V}(G)|$ and $m=|\mathrm{E}(G)|$ and let $v \in \mathrm{V}(G)$. According to Theorem 2.3(iii), $G_{v}$ is the complement of a cycle on at least 4 vertices. Since complement of any cycle with at least 6 vertices has a triangle, thus $G_{v}$ is isomorphic to $\overline{C_{4}}$ or $\overline{C_{5}}$. In particular, $n-1-\operatorname{deg}(v)=\left|\mathrm{V}\left(G_{v}\right)\right| \in\{4,5\}$, that is, either $\operatorname{deg}(v)=n-5$ or $\operatorname{deg}(v)=$ $n-6$. Assume that $a$ and $b$ are the number of vertices of $G$ with degree $n-5$ and $n-6$, respectively. Therefore $a+b=n$ and $(n-5) a+(n-6) b=2 m=n^{2}-7 n+12$ by Proposition 2.8. Solving these two equations for $a$ and $b$, we get $a=12-n$ and $b=2 n-12$. In particular, it follows that $6 \leq n \leq 12$.

Suppose that $n \geq 7$. Then as $b>0$, there exists a vertex of $G$, say $v$, with degree $n-6$. Let $A=\mathrm{N}(v)$ and $B=\mathrm{V}(G) \backslash(\mathrm{N}[v])$. By Theorem 2.3, the subgraph of $G$ induced by $B$ is $\overline{C_{5}} \cong C_{5}$ and has five edges. Also there is no edge of $G$ with both end-vertices in $\mathrm{N}(v)$, because $G$ is triangle free. Consequently, the 


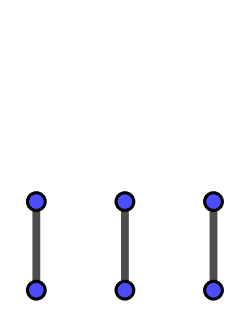

(a)

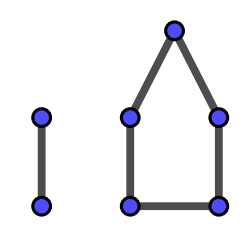

(b)

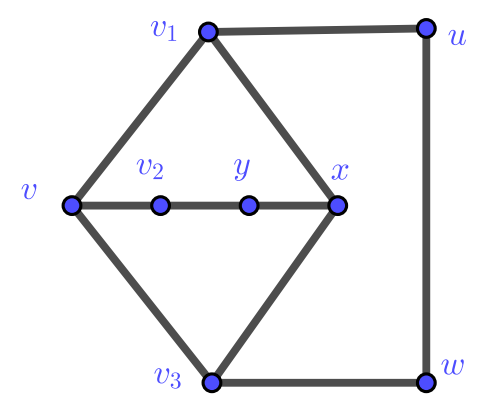

(c)

Figure 1: Triangle-free Gorenstein graphs without isolated vertex and with $\alpha=3$

subgraph of $G$ induced by $\mathrm{N}[v]$ has exactly $n-6$ edges and all the remaining edges of $G$ has one end-vertex in $A$ and one end-vertex in $B$. Assume that a vertex $x \in A$ is adjacent to at least three vertices $u_{1}, u_{2}, u_{3}$ in $B$. Since $u_{i}^{\prime}$ 's $(i=1,2,3)$ lie on a 5 -cycle, at least two of them, say $u_{1}$ and $u_{2}$, are adjacent and $x u_{1} u_{2}$ is a triangle in $G$, a contradiction. Therefore, each vertex of $A$ can be adjacent to at most two of the vertices in $B$ and the number of edges between $A$ and $B$ is at most $2|A|=$ $2(n-6)$. Hence $m \leq 2(n-6)+(n-6)+5$ and by applying Proposition 2.8, we deduce that $n^{2}-13 n+38 \leq 0$ which only holds for $n \leq 8$. We conclude that $6 \leq n \leq 8$.

If $n=6$, then $a=6$ and $b=0$, that is, $G$ consists of 6 vertices of degree one and is isomorphic to the graph (a) of Figure 1. If $n=7$, then $G$ has $a=5$ vertices of degree 2 and $b=2$ vertices of degree 1 . This means that $G$ is the disjoint union of a 5-cycle and an edge, that is, $G$ is isomorphic to Figure $1(\mathrm{~b})$.
Now assume that $n=8$ and hence $m=10$ and $G$ has $a=4$ vertices of degree 3 and $b=4$ vertices of degree 2 . Let $v$ be a vertex of degree 3. Then $G_{v}$ is $\overline{C_{4}}$ and has two edges, say $x y$ and $u w$. Assume that $\mathrm{N}(v)=\left\{v_{1}, v_{2}, v_{3}\right\}$. Since there is no edge between vertices of $\mathrm{N}(v)$ (because $G$ is trianglefree), the number of edges with one endvertex in $\mathrm{N}(v)$ and one end-vertex in $\mathrm{V}\left(G_{v}\right)$ is $m-\operatorname{deg}(v)-\left|\mathrm{E}\left(G_{v}\right)\right|=5$. Each of the four vertices of $G_{v}$ has degree at least two in $G$ and exactly one in $G_{v}$. Thus each of them is adjacent to at least one of the $v_{i}$ 's, and exactly one of the vertices of $G_{v}$, say $x$, is adjacent to two of the $v_{i}$ 's, say $v_{1}$ and $v_{3}$ (see Figure $1(\mathrm{c})$ ). So $v_{1}$ and $v_{3}$ are not adjacent to $y$ (else $v_{i} x y$ is a triangle for $i=1$ or 3 ) and $y$ must be adjacent to $v_{2}$. According to Theorem 2.3, $G_{x}$ is also isomorphic to $\overline{C_{4}}$. But $G_{x}$ is the subgraph of $G$ induced by $\left\{u, w, v, v_{2}\right\}$ and we know that $u w, v v_{2} \in \mathrm{E}\left(G_{x}\right)$. Thus there is no edge in $G$ with one end-vertex in $\{u, w\}$ and one end-vertex in $\left\{v, v_{2}\right\}$. In particular, 
$u$ and $w$ are not adjacent to $v_{2}$ and one of them say $u$ must be adjacent to $v_{1}$ and the other must be adjacent to $v_{3}$. Consequently, $G$ is the graph (c) in Figure 1.

Remark 2.10. Note that parts (iii) and (iv) of the above theorem, present a fully combinatorial characterization of Gorenstein triangle-free graphs with $\alpha=3$. Also we mention that by this theorem, the only Gorenstein triangle-free connected graph with independence number 3 is the graph (c) in Figure 1.

It is well-known that a graph is Gorenstein (over $K$ ) if and only if each of its connected components are Gorenstein (over $K$ ). Thus the above remark poses the following question.

Question 2.11. Is there an infinite family of connected Gorenstein graphs with $\alpha=3$ ?

Note that if we remove connectedness from the above question, then the family $\left\{\overline{C_{n}} \cup\right.$ $\left.K_{2} \mid 4 \leq n \in \mathbb{N}\right\}$, is an easy answer to the question.

\section{References}

[1] W. Bruns and Jürgen Herzog, CohenMacaulay Rings, Cambridge University Press, Cambridge, 1993.

[2] J. Herzog and T. Hibi, Monomial Ideals, Springer-Verlag, London , 2011.

[3] D. T. Hoang, N. C. Minh and T. N. Trung, Cohen-Macaulay graphs with large girth, J. Algebra Appl. 14:7, paper No. 1550112, 2015.

[4] D. T. Hoang and T. N. Trung, A characterization of triangle-free Gorenstein graphs and Cohen-Macaulayness of second powers of edge ideals, J. Algebr. Combin. 43, 325-338, 2016.

[5] V.E. Levit and E. Mandrescu, The independence polynomial of a graph a survey, Proceedings of the 1st International Conference on Algebraic Informatics, 233-254, Aristotle Univ. Thessaloniki, Thessaloniki, 2005.

[6] A. Nikseresht, Chordality of clutters with vertex decomposable dual and ascent of clutters, J. Combin. Theory - Ser. A, 168, 318-337, 2019, arXiv: 1708.07372.

[7] A. Nikseresht and M. R. Oboudi, Trung's construction and the CharneyDavis conjecture, Bull. Malays. Math. Sci. Soc., 2020, available online: https://doi.org/10.1007/s40840-02000933-8 .

[8] A. Nikseresht and R. Zaare-Nahandi, On generalization of cycles and chordality to clutters from an algebraic viewpoint, $\mathrm{Al}$ gebra Colloq. 24:4, 611-624, 2017.

[9] R. P. Stanley, Combinatorics and Commutative Algebra, Birkhäuser, Boston, 1996.

[10] —, Positivity problems and conjectures in algebraic combinatorics, in: V. Arnold, et al (Eds.), Mathematics: Frontiers and 
Prospectives, American Mathematical Society, pp. 295-320, 2000.

[11] J. W. Staples, On some subclasses of well-covered graphs, J. Graph Theory 3, 197-204, 1979.

[12] N. V. Trung and T. M. Tuan, Equality of ordinary and symbolic powers of Stanley-Reisner ideals, J. Algebra 328, 77-93, 2011.

[13] T. N. Trung, A characterization of Gorenstein planar graphs, in T. Hibi, ed., Adv. Stud. Pure Math., Vol. 77 (2018): The 50th Anniversary of Gröbner Bases, 399-409, arXiv:1603.00326v2. 